\title{
The van der Waals energy of atomic systems near absorbing and dispersing bodies
}

\author{
Stefan Yoshi Buhmann, Ho Trung Dung* and Dirk-Gunnar Welsch \\ Theoretisch-Physikalisches Institut, Friedrich-Schiller-Universität Jena, Max-Wien-Platz 1, 07743 Jena, Germany
}

(Dated: November 13, 2018)

\begin{abstract}
Within the frame of macroscopic quantum electrodynamics in causal media, the van der Waals interaction between an atomic system and an arbitrary arrangement of dispersing and absorbing dielectric bodies including metals is studied. It is shown that the minimal-coupling scheme and the multipolar-coupling scheme lead to essentially the same formula for the van der Waals potential. As an application, the vdW potential of an atom in the presence of a sphere is derived. Closed expressions for the long-distance (retardation) and short-distance (non-retardation) limits are given, and the effect of material absorption is discussed.
\end{abstract}

PACS numbers: $12.20 .-\mathrm{m}, 42.50 . \mathrm{Vk}, 42.50 . \mathrm{Nn}$

\section{INTRODUCTION}

The involvement of van der Waals (vdW) forces in a variety of physicochemical processes and promising potential applications (such as the construction of atomicforce microscopes [1] or reflective atom-optical elements [2]) have created the need for a very detailed understanding and controlling them. Casimir and Polder $[3]$ were the first to study the vdW interaction within the frame of rigorous quantum electrodynamics (QED). Investigating the interaction between an atom and a perfectly reflecting semi-infinite (planar) body, they found that in the shortdistance (non-retardation) limit the interaction potential $U(z)$ behaves like $z^{-3}(z$, distance between the atom and the interface), whereas in the long-distance (retardation) limit it behaves like $z^{-4}$. In their theory, Casimir and Polder quantized the (transverse) vector potential of the electromagnetic field (outside the perfectly reflecting body) in terms of normal modes and coupled them to the atom according to the minimal-coupling Hamiltonian, with its Coulomb part being determined by means of the method of image charges. They then calculated the (lowest-order) change of the ground-state energy of the system arising from this coupling, which is a function of the atomic position and thus plays the role of the potential energy that determines the force acting on the atom.

In the earlier experiments [4], which studied the deflection of thermal atomic beams by conducting surfaces, the observed signal was extremely low. Nevertheless, qualitative trends in agreement with the $z^{-3}$ law were observed. Only recent progress in experimental techniques has rendered it possible to detect $\mathrm{vdW}$ forces with sufficiently high precision [5, 6, 7, 8, 9]. In particular, by using atomic passage between two parallel plates, the $z^{-4}$ retarded potential could be verified [5]. Other methods for measuring $\mathrm{vdW}$ forces have been based on transmission

\footnotetext{
*Also at Institute of Physics, National Center for Sciences and Technology, 1 Mac Dinh Chi Street, District 1, Ho Chi Minh city, Vietnam.
}

grating diffraction of molecular beams [6], atomic quantum reflection [7], evanescent-wave atomic mirror techniques [8], and (indirect) measurements via spectroscopic means [9]. Proposals have been made on improvements of monitoring the vdW interaction by using atomic interferometry [10].

Since the appearance of Casimir's and Polder's pioneering article in 1948 there has been a large body of work on the vdW interaction (see, e.g., 11, 12, 13, 14, 15] and references therein). Roughly speaking, there have been two routes to treat the problem. In the first, which closely follows the ideas of Casimir and Polder, explicit field quantization is performed by applying standard concepts of QED, such as normal-mode techniques 16, 17, 18, 19, 20, 21, 22, 23]. In particular, extensions of the theory to one 17, 18] and two semi-infinite dielectric walls 19, 20], thin metallic films 21], and cylindrical and spherical dielectric bodies 22] have been given, and the problem of force fluctuations on short time scales has been studied [23]. The calculations have typically been based on macroscopic QED, by applying normalmode decomposition and including in it the bodies by the well-known conditions of continuity at the surfaces of discontinuity. Since in such an approach the dependence on frequency of the response to the field of the bodies cannot be properly taken into account, material dispersion and absorption are ignored. The problem does of course not occur in microscopic QED, where the bodies are treated on a microscopical level by adopting, e.g., harmonic-oscillator models (see, e.g., 17]). Apart from the fact that the calculations are rather involved, the results are model-dependent.

To overcome the difficulties mentioned, in the second route, the calculations are based on linear response theory, without (explicitly) quantizing the electromagnetic field [24, 25, 26, 27, 28, 29, 30, 31]. All the relevant entities are expressed in terms of correlation functions which in turn are related, via the fluctuation-dissipation theorem, to response functions. The method has been employed to investigate the vdW energy of an atom near a semi-infinite (planar) body made of dielectric [24, 25], metallic 26, 27], ionic-crystallic 28], and birefringent material [29]. Further, the problem of an atom near a a 
dielectric plate [26], a metallic sphere [30], and a nanowire 31] has been considered. Since the method borrows from equilibrium statistical mechanics, its applicability is restricted to the vacuum and thermal quantum states.

In this article, we give a unified QED approach to the vdW interaction between an atomic system (such as an atom or a molecule) and dispersing and absorbing dielectric bodies including metals. Starting from the quantized version of the macroscopic Maxwell field, with the medium being described in terms of a spatially varying, Kramers-Kronig consistent (complex) permittivity, we derive an expression for the vdW potential that applies to arbitrary body configurations. The formalism can be regarded as being a generalization of the normalmode formalism of macroscopic QED, so that it can be applied to other than the vacuum and thermal states, and it also allows extensions like the inclusion of dynamical interactions between the atomic system and the mediumassisted electromagnetic field. Roughly speaking, the mode expansion is replaced with some source-quantity representation in terms of the Green tensor of the macroscopic Maxwell equations, in which material dispersion and absorption are automatically included. Further, we close the gap between the minimal-coupling scheme and the multipolar-coupling scheme by showing that both approaches lead to equivalent results.

To give an application, we consider the vdW potential of an atom in the vicinity of a dispersing and absorbing microsphere. Microspheres may be interesting candidates for QED experiments (see, e.g., 32, 33 and references therein) with atomic beams. The atom-surface distances should be adjusted so that the atoms fly close enough to the surface to facilitate a strong coupling with the microsphere resonances, but not get adsorbed on the microsphere surface because of the attractive vdW force.

The article is organized as follows. In Section II the formalism is outlined and an expression for the vdW potential is derived. In Section III the formalism is applied to an atom near a sphere. A summary and some conclusions are given in Section IV

\section{THE VAN DER WAALS ENERGY}

\section{A. The quantization scheme}

Let us consider an atomic system (such as an atom or a molecule) interacting with the quantized electromagnetic field in the presence of macroscopic, dispersing and absorbing dielectric bodies. In the nonrelativistic limit, the minimal-coupling Hamiltonian in Coulomb gauge reads
34,35

$$
\begin{aligned}
\hat{H}= & \int_{0}^{\infty} \mathrm{d} \omega \hbar \omega \int \mathrm{d}^{3} r \hat{\mathbf{f}}^{\dagger}(\mathbf{r}, \omega) \hat{\mathbf{f}}(\mathbf{r}, \omega) \\
& +\sum_{\alpha} \frac{1}{2 m_{\alpha}}\left[\hat{\mathbf{p}}_{\alpha}-q_{\alpha} \hat{\mathbf{A}}\left(\mathbf{r}_{\mathrm{A}}+\hat{\mathbf{r}}_{\alpha}\right)\right]^{2} \\
& +\frac{1}{2} \int \mathrm{d}^{3} r \hat{\rho}_{A}(\mathbf{r}) \hat{\varphi}_{\mathrm{A}}(\mathbf{r})+\int \mathrm{d}^{3} r \hat{\rho}_{A}(\mathbf{r}) \hat{\varphi}_{\mathrm{M}}(\mathbf{r}),
\end{aligned}
$$

where $m_{\alpha}$ and $q_{\alpha}$ are respectively the masses and charges of the particles constituting the atomic system, while $\hat{\mathbf{r}}_{\alpha}$ and $\hat{\mathbf{p}}_{\alpha}$ are respectively their coordinates (relative to the center of mass $\mathbf{r}_{\mathrm{A}}$ ) and canonically conjugated momenta. The first term in the Hamiltonian describes the combined system of the electromagnetic field plus the macroscopic bodies (including dissipative systems) in terms of bosonic vector fields $\hat{\mathbf{f}}(\mathbf{r}, \omega)$, which satisfy the commutation relations

$$
\begin{array}{r}
{\left[\hat{f}_{i}(\mathbf{r}, \omega), \hat{f}_{j}^{\dagger}\left(\mathbf{r}^{\prime}, \omega^{\prime}\right)\right]=\delta_{i j} \delta\left(\mathbf{r}-\mathbf{r}^{\prime}\right) \delta\left(\omega-\omega^{\prime}\right),} \\
{\left[\hat{f}_{i}(\mathbf{r}, \omega), \hat{f}_{j}\left(\mathbf{r}^{\prime}, \omega^{\prime}\right)\right]=\left[\hat{f}_{i}^{\dagger}(\mathbf{r}, \omega), \hat{f}_{j}^{\dagger}\left(\mathbf{r}^{\prime}, \omega^{\prime}\right)\right]=0 .}
\end{array}
$$

The second term is the kinetic energy of the charged particles, while the third term describes their mutual Coulomb interaction, where

$$
\hat{\varphi}_{\mathrm{A}}(\mathbf{r})=\frac{1}{4 \pi \varepsilon_{0}} \int \mathrm{d}^{3} r^{\prime} \frac{\hat{\rho}_{\mathrm{A}}\left(\mathbf{r}^{\prime}\right)}{\left|\mathbf{r}-\mathbf{r}^{\prime}\right|}
$$

and

$$
\hat{\rho}_{\mathrm{A}}(\mathbf{r})=\sum_{\alpha} q_{\alpha} \delta\left[\mathbf{r}-\left(\mathbf{r}_{\mathrm{A}}+\hat{\mathbf{r}}_{\alpha}\right)\right]
$$

are respectively the scalar potential and the charge density of the atomic system. The last term accounts for the Coulomb interaction of the particles with the medium. The vector potential $\hat{\mathbf{A}}(\mathbf{r})$ and the scalar potential $\hat{\varphi}_{\mathrm{M}}(\mathbf{r})$ of the medium-assisted electromagnetic field are given by

$$
\begin{gathered}
\hat{\mathbf{A}}(\mathbf{r})=\int_{0}^{\infty} \mathrm{d} \omega(i \omega)^{-1} \underline{\hat{\mathbf{E}}}^{\perp}(\mathbf{r}, \omega)+\text { H.c. }, \\
-\boldsymbol{\nabla} \hat{\varphi}_{\mathrm{M}}(\mathbf{r})=\int_{0}^{\infty} \mathrm{d} \omega \underline{\hat{\mathbf{E}}}^{\|}(\mathbf{r}, \omega)+\text { H.c. }
\end{gathered}
$$

where the symbols $\perp$ and $\|$ are used to distinguish transverse and longitudinal vector fields, respectively. In particular, $\underline{\hat{\mathbf{E}}}^{\perp}(\mathbf{r}, \omega)$ and $\underline{\hat{\mathbf{E}}}^{\|}(\mathbf{r}, \omega)$ read

$$
\underline{\hat{\mathbf{E}}}^{\perp(\|)}(\mathbf{r}, \omega)=\int \mathrm{d}^{3} r^{\prime} \boldsymbol{\delta}^{\perp(\|)}\left(\mathbf{r}-\mathbf{r}^{\prime}\right) \underline{\hat{\mathbf{E}}}\left(\mathbf{r}^{\prime}, \omega\right),
$$

where $\boldsymbol{\delta}^{\perp}(\mathbf{r})$ and $\boldsymbol{\delta}^{\|}(\mathbf{r})$ are the transverse and longitudinal dyadic $\delta$-functions, respectively, and the mediumassisted electric field in the $\omega$-domain, $\underline{\hat{\mathbf{E}}}(\mathbf{r}, \omega)$, is expressed in terms of the basic variables $\hat{\mathbf{f}}(\mathbf{r}, \omega)$ as

$$
\begin{aligned}
\hat{\mathbf{E}}(\mathbf{r}, \omega)=i \sqrt{\frac{\hbar}{\pi \varepsilon_{0}}} \frac{\omega^{2}}{c^{2}} \int & \mathrm{d}^{3} r^{\prime} \sqrt{\operatorname{Im} \varepsilon\left(\mathbf{r}^{\prime}, \omega\right)} \\
& \times \boldsymbol{G}\left(\mathbf{r}, \mathbf{r}^{\prime}, \omega\right) \hat{\mathbf{f}}\left(\mathbf{r}^{\prime}, \omega\right)
\end{aligned}
$$


$\left(c=1 / \sqrt{\varepsilon_{0} \mu_{0}}\right)$. Here, $\boldsymbol{G}\left(\mathbf{r}, \mathbf{r}^{\prime}, \omega\right)$ is the classical Green tensor and $\operatorname{Im} \varepsilon(\mathbf{r}, \omega)$ is the imaginary part of the complex, space- and frequency-dependent (relative) permittivity

$$
\varepsilon(\mathbf{r}, \omega)=\operatorname{Re} \varepsilon(\mathbf{r}, \omega)+i \operatorname{Im} \varepsilon(\mathbf{r}, \omega)
$$

The Green tensor, which obeys the inhomogeneous partial differential equation

$$
\left[\boldsymbol{\nabla} \times \nabla \times-\frac{\omega^{2}}{c^{2}} \varepsilon(\mathbf{r}, \omega)\right] \boldsymbol{G}\left(\mathbf{r}, \mathbf{r}^{\prime}, \omega\right)=\boldsymbol{\delta}\left(\mathbf{r}-\mathbf{r}^{\prime}\right)
$$

together with the boundary condition at infinity, has the following general properties (see, e.g., 35]):

$$
\begin{gathered}
\boldsymbol{G}^{*}\left(\mathbf{r}, \mathbf{r}^{\prime}, \omega\right)=\boldsymbol{G}\left(\mathbf{r}, \mathbf{r}^{\prime},-\omega^{*}\right) \\
G_{i j}\left(\mathbf{r}, \mathbf{r}^{\prime}, \omega\right)=G_{j i}\left(\mathbf{r}^{\prime}, \mathbf{r}, \omega\right), \\
\frac{\omega^{2}}{c^{2}} \int \mathrm{d}^{3} s \varepsilon_{\mathrm{I}}(\mathbf{s}, \omega) \boldsymbol{G}(\mathbf{r}, \mathbf{s}, \omega) \boldsymbol{G}^{*}\left(\mathbf{s}, \mathbf{r}^{\prime}, \omega\right)=\operatorname{Im} \boldsymbol{G}\left(\mathbf{r}, \mathbf{r}^{\prime}, \omega\right) .
\end{gathered}
$$

In this way, all electromagnetic-field quantities can be expressed in terms of the fundamental fields $\hat{\mathbf{f}}(\mathbf{r}, \omega)$. In particular, the operator for the electric field reads

$$
\begin{aligned}
\hat{\mathbf{E}}(\mathbf{r}) & =-\frac{1}{i \hbar}[\hat{\mathbf{A}}(\mathbf{r}), \hat{H}]-\boldsymbol{\nabla} \hat{\varphi}_{\mathrm{M}}(\mathbf{r})-\boldsymbol{\nabla} \hat{\varphi}_{\mathrm{A}}(\mathbf{r}) \\
& =\hat{\mathbf{E}}_{\mathrm{M}}(\mathbf{r})+\sum_{\alpha} \frac{q_{\alpha}\left[\mathbf{r}-\left(\mathbf{r}_{\mathrm{A}}+\hat{\mathbf{r}}_{\alpha}\right)\right]}{4 \pi \varepsilon_{0}\left|\mathbf{r}-\left(\mathbf{r}_{\mathrm{A}}+\hat{\mathbf{r}}_{\alpha}\right)\right|^{3}}
\end{aligned}
$$

where

$$
\hat{\mathbf{E}}_{\mathrm{M}}(\mathbf{r})=\int_{0}^{\infty} \mathrm{d} \omega \underline{\hat{\mathbf{E}}}(\mathbf{r}, \omega)+\text { H.c. }
$$

and the polarization field associated with the dielectric medium is given by

$$
\hat{\mathbf{P}}_{\mathrm{M}}(\mathbf{r})=\int_{0}^{\infty} \mathrm{d} \omega \underline{\hat{\mathbf{P}}}(\mathbf{r}, \omega)+\text { H.c. },
$$

where

$$
\underline{\hat{\mathbf{P}}}(\mathbf{r}, \omega)=\chi(\mathbf{r}, \omega) \varepsilon_{0} \underline{\hat{\mathbf{E}}}(\mathbf{r}, \omega)+\hat{\mathbf{P}}_{\mathrm{N}}(\mathbf{r}, \omega) .
$$

Here,

$$
\chi(\mathbf{r}, \omega)=\varepsilon(\mathbf{r}, \omega)-1
$$

is the dielectric susceptibility and

$$
\hat{\mathbf{P}}_{\mathrm{N}}(\mathbf{r}, \omega)=i \sqrt{\frac{\hbar \varepsilon_{0}}{\pi} \operatorname{Im} \varepsilon(\mathbf{r}, \omega)} \hat{\mathbf{f}}(\mathbf{r}, \omega)
$$

is the so-called noise polarization.
For the following it is convenient to decompose the Hamiltonian (1) as

$$
\hat{H}=\hat{H}_{\mathrm{F}}+\hat{H}_{\mathrm{A}}+\hat{H}_{\mathrm{AF}},
$$

where

$$
\begin{aligned}
\hat{H}_{\mathrm{F}} & \equiv \int_{0}^{\infty} \mathrm{d} \omega \hbar \omega \int \mathrm{d}^{3} r \hat{\mathbf{f}}^{\dagger}(\mathbf{r}, \omega) \hat{\mathbf{f}}(\mathbf{r}, \omega) \\
\hat{H}_{\mathrm{A}} & \equiv \sum_{\alpha} \frac{\hat{\mathbf{p}}_{\alpha}^{2}}{2 m_{\alpha}}+\frac{1}{2} \int \mathrm{d}^{3} r \hat{\rho}_{\mathrm{A}}(\mathbf{r}) \hat{\varphi}_{\mathrm{A}}(\mathbf{r}) \\
& =\sum_{\alpha} \frac{\hat{\mathbf{p}}_{\alpha}^{2}}{2 m_{\alpha}}+\sum_{\alpha<\beta} \frac{q_{\alpha} q_{\beta}}{4 \pi \varepsilon_{0}\left|\hat{\mathbf{r}}_{\alpha}-\hat{\mathbf{r}}_{\beta}\right|} \\
\hat{H}_{\mathrm{AF}} \equiv & \int \mathrm{d}^{3} r \hat{\rho}_{A}(\mathbf{r}) \hat{\varphi}_{\mathrm{M}}(\mathbf{r})-\sum_{\alpha} \frac{q_{\alpha}}{m_{\alpha}} \hat{\mathbf{A}}\left(\mathbf{r}_{\mathrm{A}}+\hat{\mathbf{r}}_{\alpha}\right) \\
& \times\left[\hat{\mathbf{p}}_{\alpha}-\frac{1}{2} q_{\alpha} \hat{\mathbf{A}}\left(\mathbf{r}_{\mathrm{A}}+\hat{\mathbf{r}}_{\alpha}\right)\right]
\end{aligned}
$$

Obviously, $\hat{H}_{\mathrm{F}}$ is the Hamiltonian of the medium-assisted electromagnetic field, $\hat{H}_{\mathrm{A}}$ is the Hamiltonian of the atomic system with eigenstates $|n\rangle$ and eigenvalues $E_{n}$ according to

$$
\hat{H}_{\mathrm{A}}|n\rangle=E_{n}|n\rangle
$$

and $\hat{H}_{\mathrm{AF}}$ is the interaction energy between them. For a neutral atomic system in the electric-dipole approximation, the latter simplifies to

$$
\hat{H}_{\mathrm{AF}}=\hat{H}_{\mathrm{AF}}^{(\mathrm{I})}+\hat{H}_{\mathrm{AF}}^{(\mathrm{II})}
$$

$$
\hat{H}_{\mathrm{AF}}^{(\mathrm{I})} \equiv-\sum_{\alpha} \frac{q_{\alpha}}{m_{\alpha}} \hat{\mathbf{p}}_{\alpha} \hat{\mathbf{A}}\left(\mathbf{r}_{\mathrm{A}}\right)+\hat{\mathbf{d}} \nabla \hat{\varphi}_{\mathrm{M}}\left(\mathbf{r}_{\mathrm{A}}\right)
$$

$$
\hat{H}_{\mathrm{AF}}^{(\mathrm{II})} \equiv \sum_{\alpha} \frac{q_{\alpha}^{2}}{2 m_{\alpha}} \hat{\mathbf{A}}^{2}\left(\mathbf{r}_{\mathrm{A}}\right)
$$

where $\hat{\mathbf{d}}$ is the electric dipole operator of the atomic system

$$
\hat{\mathbf{d}}=\sum_{\alpha} q_{\alpha} \hat{\mathbf{r}}_{\alpha}
$$

\section{B. The vdW energy in the minimal-coupling scheme}

Following the original line of Casimir and Polder [3], we calculate the vdW energy of an atomic system as the position-dependent part of the leading-order correction to the unperturbed ground state energy due to the perturbation according to Eq. (26) [together with Eqs. (27) 
and (28)]. Since the diagonal matrix elements of $\hat{H}_{\mathrm{AF}}^{(\mathrm{I})}$, Eq. (27), are zero, the non-vanishing contribution to the energy correction from first-order perturbation theory is due to $\hat{H}_{\mathrm{AF}}^{(\mathrm{II})}$, Eq. (28). Inspection of Eq. (28) shows that this contribution is of linear order in the fine structure constant $\alpha=e^{2} /\left(2 \varepsilon_{0} \hbar c\right)$. We have thus to include that contribution from second-order perturbation theory which is of the same order in $\alpha$. Therefore, we apply first-order perturbation theory for $\hat{H}_{\mathrm{AF}}^{(\mathrm{II})}$ and second-order perturbation theory for $\hat{H}_{\mathrm{AF}}^{(\mathrm{I})}$. The energy correction to the ground state thus reads

$$
\Delta E \simeq \Delta_{1} E+\Delta_{2} E
$$

where

$$
\begin{aligned}
\Delta_{1} E & \equiv\left\langle 0\left|\left\langle\{0\}\left|\hat{H}_{A F}^{(\mathrm{II})}\right|\{0\}\right\rangle\right| 0\right\rangle \\
& =\left\langle 0\left|\left\langle\{0\}\left|\sum_{\alpha} \frac{q_{\alpha}^{2}}{2 m_{\alpha}} \hat{\mathbf{A}}^{2}\left(\mathbf{r}_{\mathrm{A}}\right)\right|\{0\}\right\rangle\right| 0\right\rangle
\end{aligned}
$$

and

$$
\begin{aligned}
& \Delta_{2} E \equiv \sum_{n} \int_{0}^{\infty} \mathrm{d} \omega \int \mathrm{d}^{3} r \frac{\left|\left\langle 0\left|\left\langle\{0\}\left|\hat{H}_{\mathrm{AF}}^{(\mathrm{I})}\right|\{\mathbf{1}(\mathbf{r}, \omega)\}\right\rangle\right| n\right\rangle\right|^{2}}{E_{0}-\left(E_{n}+\hbar \omega\right)} \\
& =\frac{1}{\hbar} \sum_{n} \int_{0}^{\infty} \frac{\mathrm{d} \omega}{\omega_{n}+\omega} \int \mathrm{d}^{3} r \mid\langle 0|\langle\{0\}| \sum_{\alpha} \frac{q_{\alpha}}{m_{\alpha}} \hat{\mathbf{p}}_{\alpha} \hat{\mathbf{A}}\left(\mathbf{r}_{\mathrm{A}}\right) \\
& -\left.\hat{\mathbf{d}} \nabla \hat{\varphi}_{\mathrm{M}}\left(\mathbf{r}_{\mathrm{A}}\right)|\{\mathbf{1}(\mathbf{r}, \omega)\}\rangle|n\rangle\right|^{2} .
\end{aligned}
$$

Here, $|0\rangle$ and $|\{0\}\rangle$ are respectively the ground state of the atomic system and the ground state of the mediumassisted electromagnetic field, and

$$
|\{\mathbf{1}(\mathbf{r}, \omega)\}\rangle \equiv \hat{\mathbf{f}}^{\dagger}(\mathbf{r}, \omega)|\{0\}\rangle
$$

denotes single-quantum Fock states of the fundamental fields. Further,

$$
\omega_{n}=\left(E_{n}-E_{0}\right) / \hbar
$$

are the transition frequencies between the excited atomic states and the ground state. Note that due to the linear dependence of the vector potential and the gradient of the scalar potential on $\hat{\mathbf{f}}(\mathbf{r}, \omega)$ [and $\left.\hat{\mathbf{f}}^{\dagger}(\mathbf{r}, \omega)\right]$, the nonvanishing matrix elements of $\hat{H}_{\mathrm{AF}}^{(\mathrm{I})}$ in the Fock-state basis (which is defined as the set of eigenvectors of $\hat{H}_{\mathrm{F}}$ ) are those between Fock states that just differ in one quantum.

Expressing in Eq. (31) $\hat{\mathbf{A}}\left(\mathbf{r}_{\mathrm{A}}\right)$ in terms of $\hat{\mathbf{f}}(\mathbf{r})$ [and $\hat{\mathbf{f}}^{\dagger}(\mathbf{r})$ ], on using Eqs. (6), (8), and (9), recalling Eq. (33), and exploiting the integral relation (14) for the contraction of two Green tensors, we derive after some lengthy but straightforward calculation

$$
\Delta_{1} E=\frac{\hbar \mu_{0}}{\pi} \sum_{\alpha} \frac{q_{\alpha}^{2}}{2 m_{\alpha}} \int_{0}^{\infty} \mathrm{d} \omega \operatorname{Im}^{\perp} G_{i i}^{\perp}\left(\mathbf{r}_{\mathrm{A}}, \mathbf{r}_{\mathrm{A}}, \omega\right),
$$

where

$$
\begin{aligned}
& { }^{\perp(\|)} \boldsymbol{G}^{\perp(\|)}\left(\mathbf{r}, \mathbf{r}^{\prime}, \omega\right) \\
& \quad \equiv \int \mathrm{d}^{3} s \int \mathrm{d}^{3} s^{\prime} \boldsymbol{\delta}^{\perp(\|)}(\mathbf{r}-\mathbf{s}) \boldsymbol{G}\left(\mathbf{s}, \mathbf{s}^{\prime}, \omega\right) \boldsymbol{\delta}^{\perp(\|)}\left(\mathbf{s}^{\prime}-\mathbf{r}^{\prime}\right) .
\end{aligned}
$$

In Eq. (35) and below, summation over repeated vector indices is understood. Using the sum rule

$$
\sum_{\alpha} \frac{q_{\alpha}^{2}}{2 m_{\alpha}} \delta_{i j}=\frac{1}{2 \hbar} \sum_{n} \omega_{n}\left(d_{0 n, i} d_{n 0, j}+d_{0 n, j} d_{n 0, i}\right)
$$

(for a proof, see Appendix $\mathrm{A}$ ), where

$$
d_{0 n, i} \equiv\left\langle 0\left|\hat{d}_{i}\right| n\right\rangle,
$$

denote the matrix elements of the electric-dipole operator (29), we may equivalently represent Eq. (35) in the form of

$$
\begin{gathered}
\Delta_{1} E=\frac{\hbar \mu_{0}}{\pi} \sum_{\alpha} \frac{q_{\alpha}^{2}}{2 m_{\alpha}} \delta_{i j} \int_{0}^{\infty} \mathrm{d} \omega \operatorname{Im}^{\perp} G_{i j}^{\perp}\left(\mathbf{r}_{\mathrm{A}}, \mathbf{r}_{\mathrm{A}}, \omega\right) \\
=\frac{\mu_{0}}{\pi} \sum_{n} \int_{0}^{\infty} \mathrm{d} \omega \omega_{n} \mathbf{d}_{0 n} \operatorname{Im}^{\perp} G^{\perp}\left(\mathbf{r}_{\mathrm{A}}, \mathbf{r}_{\mathrm{A}}, \omega\right) \mathbf{d}_{n 0} .
\end{gathered}
$$

In a similar fashion, we find that Eq. (32) leads to

$$
\begin{aligned}
& \Delta_{2} E=-\frac{\mu_{0}}{\pi} \sum_{n} \int_{0}^{\infty} \mathrm{d} \omega\left[\frac{\omega_{n}^{2}}{\omega_{n}+\omega} \int \mathrm{d}^{3} s\right. \\
& \left.\times \int \mathrm{d}^{3} s^{\prime} \mathbf{d}_{0 n} \boldsymbol{\mu}(\mathbf{s}, \omega) \operatorname{Im} \boldsymbol{G}\left(\mathbf{s}, \mathbf{s}^{\prime}, \omega\right) \boldsymbol{\mu}\left(\mathbf{s}^{\prime}, \omega\right) \mathbf{d}_{n 0}\right],
\end{aligned}
$$

where, on using the relation (A3), the matrix elements of the electric-dipole moment (38) have been introduced and the abbreviating notation

$$
\boldsymbol{\mu}(\mathbf{r}, \omega) \equiv \boldsymbol{\delta}^{\perp}\left(\mathbf{r}-\mathbf{r}_{\mathrm{A}}\right)-\frac{\omega}{\omega_{n}} \boldsymbol{\delta}^{\|}\left(\mathbf{r}-\mathbf{r}_{\mathrm{A}}\right)
$$

has been used.

We substitute Eqs. (39) and (40) into Eq. (30), and obtain, on recalling Eq. (41),

$$
\begin{aligned}
\Delta E=- & \frac{\mu_{0}}{\pi} \sum_{n} \int_{0}^{\infty} \frac{\mathrm{d} \omega}{\omega_{n}+\omega} \mathbf{d}_{0 n}\left\{\omega^{2} \operatorname{Im}^{\|} \boldsymbol{G}^{\|}\left(\mathbf{r}_{\mathrm{A}}, \mathbf{r}_{\mathrm{A}}, \omega\right)\right. \\
& -\omega_{n} \omega\left[\operatorname{Im}^{\perp} \boldsymbol{G}^{\perp}\left(\mathbf{r}_{\mathrm{A}}, \mathbf{r}_{\mathrm{A}}, \omega\right)+\operatorname{Im}^{\perp} \boldsymbol{G}^{\|}\left(\mathbf{r}_{\mathrm{A}}, \mathbf{r}_{\mathrm{A}}, \omega\right)\right. \\
& \left.\left.+\operatorname{Im}^{\|} \boldsymbol{G}^{\perp}\left(\mathbf{r}_{\mathrm{A}}, \mathbf{r}_{\mathrm{A}}, \omega\right)\right]\right\} \mathbf{d}_{n 0} .
\end{aligned}
$$

Let $\mathcal{R}$ be the (small) region of space where the atom is situated and the permittivity can be regarded as being effectively not varying with space, i.e., $\varepsilon(\mathbf{r}, \omega) \simeq \varepsilon\left(\mathbf{r}_{\mathrm{A}}, \omega\right)$ if $\mathbf{r} \in \mathcal{R}$. For $\mathbf{r}, \mathbf{r}^{\prime} \in \mathcal{R}$, the Green tensor can then be given in the form

$$
\boldsymbol{G}\left(\mathbf{r}, \mathbf{r}^{\prime}, \omega\right)=\boldsymbol{G}^{(0)}\left(\mathbf{r}, \mathbf{r}^{\prime}, \omega\right)+\boldsymbol{G}^{(1)}\left(\mathbf{r}, \mathbf{r}^{\prime}, \omega\right)
$$


with $\boldsymbol{G}^{(0)}\left(\mathbf{r}, \mathbf{r}^{\prime}, \omega\right)$ denoting the (translationally invariant) bulk Green tensor that corresponds to $\varepsilon\left(\mathbf{r}_{\mathrm{A}}, \omega\right)$ and $\boldsymbol{G}^{(1)}\left(\mathbf{r}, \mathbf{r}^{\prime}, \omega\right)$ being the scattering Green tensor that accounts for the spatial variation of the permittivity. In practice, the atom is typically situated in a free-space region, so that $\boldsymbol{G}^{(0)}$ is simply the vacuum Green tensor. According to the decomposition of the Green tensor in Eq. (43), the energy correction $\Delta E$ given by Eq. (42) consists of two terms,

$$
\Delta E=\Delta E^{(0)}+\Delta E^{(1)}\left(\mathbf{r}_{\mathrm{A}}\right),
$$

where the $\mathbf{r}_{\mathrm{A}}$-independent term $\Delta E^{(0)}$, which is related to the bulk Green tensor, gives rise to the (vacuum) Lamb shift, whereas the $\mathbf{r}_{\mathrm{A}}$-dependent term $\Delta E^{(1)}\left(\mathbf{r}_{\mathrm{A}}\right)$, which is related to the scattering Green tensor, is just the $\mathrm{vdW}$ energy sought:

$$
\begin{aligned}
& U\left(\mathbf{r}_{\mathrm{A}}\right) \equiv \Delta E^{(1)}\left(\mathbf{r}_{\mathrm{A}}\right)=-\frac{\mu_{0}}{\pi} \sum_{n} \int_{0}^{\infty} \frac{\mathrm{d} \omega}{\omega_{n}+\omega} \mathbf{d}_{0 n} \\
& \times\left\{\omega^{2} \operatorname{Im}^{\|} \boldsymbol{G}^{(1) \|}\left(\mathbf{r}_{\mathrm{A}}, \mathbf{r}_{\mathrm{A}}, \omega\right)-\omega_{n} \omega\right. \\
& \times\left[\operatorname{Im}^{\perp} \boldsymbol{G}^{(1) \perp}\left(\mathbf{r}_{\mathrm{A}}, \mathbf{r}_{\mathrm{A}}, \omega\right)+\operatorname{Im}^{\perp} \boldsymbol{G}^{(1) \|}\left(\mathbf{r}_{\mathrm{A}}, \mathbf{r}_{\mathrm{A}}, \omega\right)\right. \\
& \left.\left.\quad+\operatorname{Im}^{\|} \boldsymbol{G}^{(1) \perp}\left(\mathbf{r}_{\mathrm{A}}, \mathbf{r}_{\mathrm{A}}, \omega\right)\right]\right\} \mathbf{d}_{n 0} .
\end{aligned}
$$

To further evaluate this expression, it is convenient to express it in terms of the whole scattering Green tensor rather than its imaginary part. For this purpose, we write $\operatorname{Im} \boldsymbol{G}^{(1)}=\left(\boldsymbol{G}^{(1)}-\boldsymbol{G}^{(1) *}\right) /(2 i)$, recall the relation (12), and change the integration variable from $-\omega$ to $\omega$. Equation (45) then changes to

$$
\begin{aligned}
U\left(\mathbf{r}_{\mathrm{A}}\right)= & \frac{\mu_{0}}{2 i \pi} \sum_{n} \mathbf{d}_{0 n}\left(\int _ { 0 } ^ { \infty } \frac { \mathrm { d } \omega } { \omega _ { n } + \omega } \left\{\omega_{n} \omega\left[{ }^{\perp} \boldsymbol{G}^{(1) \perp}\left(\mathbf{r}_{\mathrm{A}}, \mathbf{r}_{\mathrm{A}}, \omega\right)+{ }^{\perp} \boldsymbol{G}^{(1) \|}\left(\mathbf{r}_{\mathrm{A}}, \mathbf{r}_{\mathrm{A}}, \omega\right)+{ }^{\|} \boldsymbol{G}^{(1) \perp}\left(\mathbf{r}_{\mathrm{A}}, \mathbf{r}_{\mathrm{A}}, \omega\right)\right]\right.\right. \\
& \left.-\omega^{2 \|} \boldsymbol{G}^{(1) \|}\left(\mathbf{r}_{\mathrm{A}}, \mathbf{r}_{\mathrm{A}}, \omega\right)\right\}+\int_{-\infty}^{0} \frac{\mathrm{d} \omega}{\omega_{n}-\omega}\left\{\omega_{n} \omega\left[{ }^{\perp} \boldsymbol{G}^{(1) \perp}\left(\mathbf{r}_{\mathrm{A}}, \mathbf{r}_{\mathrm{A}}, \omega\right)+{ }^{\perp} \boldsymbol{G}^{(1) \|}\left(\mathbf{r}_{\mathrm{A}}, \mathbf{r}_{\mathrm{A}}, \omega\right)+{ } \boldsymbol{G}^{(1) \perp}\left(\mathbf{r}_{\mathrm{A}}, \mathbf{r}_{\mathrm{A}}, \omega\right)\right]\right. \\
& \left.\left.+\omega^{2 \|} \boldsymbol{G}^{(1) \|}\left(\mathbf{r}_{\mathrm{A}}, \mathbf{r}_{\mathrm{A}}, \omega\right)\right\}\right) \mathbf{d}_{n 0} .
\end{aligned}
$$

This equation can be greatly simplified by using contourintegral techniques. Note that $\boldsymbol{G}^{(1)}\left(\mathbf{r}_{\mathrm{A}}, \mathbf{r}_{\mathrm{A}}, \omega\right)$ is an analytic function in the upper complex half plane $(\operatorname{Im} \omega \geq 0$, $\omega \neq 0)$. Further, ${ }^{\perp} \boldsymbol{G}^{(1) \perp}\left(\mathbf{r}_{\mathrm{A}}, \mathbf{r}_{\mathrm{A}}, \omega\right),{ }^{\perp} \boldsymbol{G}^{(1) \|}\left(\mathbf{r}_{\mathrm{A}}, \mathbf{r}_{\mathrm{A}}, \omega\right)$, and ${ }^{\|} \boldsymbol{G}^{(1) \perp}\left(\mathbf{r}_{\mathrm{A}}, \mathbf{r}_{\mathrm{A}}, \omega\right)$ tend to zero as $\omega$ approaches zero, because the asymptote of the Green tensor contains no transverse components [cf. Eq. (B14)]. Finally, the term $\omega^{2} \| \boldsymbol{G}^{(1) \|}\left(\mathbf{r}_{\mathrm{A}}, \mathbf{r}_{\mathrm{A}}, \omega\right)$ is also well-behaved for vanishing $\omega$, as can be seen from Eq. (B7). Consequently, the integrands of the $\omega$-integrals in Eq. (46) are analytic functions without poles in the whole upper complex half plane, including the real axis. We may therefore apply Cauchy's theorem, and replace the integral over the positive (negative) real half axis by a contour integral along the positive imaginary half axis (introducing the purely imaginary coordinate $\omega=i u$ ) and along a quarter circle with infinite radius in the first (second) quadrant of the complex frequency plane. Since the integrals along the infinitely large quarter circles vanish [cf. Eq. [B3 $]$, we finally arrive at

$$
U\left(\mathbf{r}_{\mathrm{A}}\right)=\frac{\mu_{0}}{\pi} \sum_{n} \int_{0}^{\infty} \mathrm{d} u \frac{\omega_{n} u^{2}}{\omega_{n}^{2}+u^{2}} \mathbf{d}_{0 n} \boldsymbol{G}^{(1)}\left(\mathbf{r}_{\mathrm{A}}, \mathbf{r}_{\mathrm{A}}, i u\right) \mathbf{d}_{n 0},
$$

where the identity $\boldsymbol{G}^{(1)}={ }^{\perp} \boldsymbol{G}^{(1) \perp}+{ }^{\perp} \boldsymbol{G}^{(1) \|}+{ }^{\|} \boldsymbol{G}^{(1) \perp}+$ $\| \boldsymbol{G}^{(1) \|}$ has been taken into account.

Introducing the (lowest-order) ground-state polarizability tensor

$$
\boldsymbol{\alpha}(\omega)=\lim _{\eta \rightarrow 0+} \frac{2}{\hbar} \sum_{n} \frac{\omega_{n}}{\omega_{n}^{2}-\omega^{2}-i \eta \omega} \mathbf{d}_{0 n} \otimes \mathbf{d}_{n 0}
$$

of the atomic system (see, e.g., [36]), we may represent Eq. (47) in the equivalent form of

$$
U\left(\mathbf{r}_{\mathrm{A}}\right)=\frac{\hbar \mu_{0}}{2 \pi} \int_{0}^{\infty} \mathrm{d} u u^{2} \alpha_{i j}(i u) G_{i j}^{(1)}\left(\mathbf{r}_{\mathrm{A}}, \mathbf{r}_{\mathrm{A}}, i u\right) .
$$


It is worth noting that Eq. (49) directly follows from QED in causal media, without the need of additional assumptions borrowed from other fields. Equation (49) expresses the vdW potential of an arbitrary atomic system (such as an atom or a molecule) in the presence of an arbitrary configuration of dispersing and absorbing macroscopic dielectric bodies in terms of the polarizability tensor of the atomic system in lowest order of perturbation theory and the scattering Green tensor of the macroscopic Maxwell equations.

In particular for an atom, one can make use of the spherical symmetry and reduce Eq. (49) to

$$
U\left(\mathbf{r}_{\mathrm{A}}\right)=\frac{\hbar \mu_{0}}{2 \pi} \int_{0}^{\infty} \mathrm{d} u u^{2} \alpha(i u) G_{i i}^{(1)}\left(\mathbf{r}_{\mathrm{A}}, \mathbf{r}_{\mathrm{A}}, i u\right)
$$

where

$$
\alpha(\omega)=\lim _{\eta \rightarrow 0+} \frac{2}{3 \hbar} \sum_{n} \frac{\omega_{n}}{\omega_{n}^{2}-\omega^{2}-i \eta \omega}\left|\mathbf{d}_{0 n}\right|^{2} .
$$

This result agrees with the results inferred from (semi-)classical linear response theory. Note that the field susceptibility introduced in Refs. 24] and 26] differs from the scattering Green tensor by a factor of $\omega^{2}$. Needless to say, that in the special case of a two-level atom, Eq. (50) reduces to the result, e.g., in Ref. 25]. The derivation of Eq. (50) shows that it can be regarded as the natural extension of the QED results obtained on the basis of the normal-mode formalism, which ignores material absorption.

\section{The vdW energy in the multipolar-coupling scheme}

Let us turn to the multipolar-coupling scheme widely used for studying the interaction of electromagnetic fields with atoms and molecules. Just as in standard QED, so in the present formalism the multipolar-coupling Hamiltonian can be obtained from the minimal-coupling Hamiltonian by means of a Power-Zienau transformation,

$$
\hat{\mathcal{H}}=\hat{U}^{\dagger} \hat{H} \hat{U}
$$

where

$$
\hat{U}=\exp \left[\frac{i}{\hbar} \int \mathrm{d}^{3} r \hat{\mathbf{P}}_{\mathrm{A}}(\mathbf{r}) \hat{\mathbf{A}}(\mathbf{r})\right]
$$

with

$$
\hat{\mathbf{P}}_{\mathrm{A}}(\mathbf{r})=\sum_{\alpha} q_{\alpha} \hat{\mathbf{r}}_{\alpha} \int_{0}^{1} \mathrm{~d} \lambda \delta\left[\mathbf{r}-\left(\mathbf{r}_{\mathrm{A}}+\lambda \hat{\mathbf{r}}_{\alpha}\right)\right]
$$

being the polarization associated with the (neutral) atomic system. Using $\hat{H}$ from Eq. (11), we derive [35, 37]

$$
\begin{aligned}
\hat{\mathcal{H}} & =\int \mathrm{d}^{3} r \int_{0}^{\infty} \mathrm{d} \omega \hbar \omega \hat{\mathbf{f}}^{\dagger}(\mathbf{r}, \omega) \hat{\mathbf{f}}(\mathbf{r}, \omega)+\sum_{\alpha} \frac{1}{2 m_{\alpha}}\left\{\hat{\mathbf{p}}_{\alpha}\right. \\
& \left.+q_{\alpha} \int_{0}^{1} \mathrm{~d} \lambda \lambda \hat{\mathbf{r}}_{\alpha} \times \hat{\mathbf{B}}\left[\mathbf{r}_{\mathrm{A}}+\lambda \hat{\mathbf{r}}_{\alpha}\right]\right\}^{2} \\
& +\frac{1}{2 \varepsilon_{0}} \int \mathrm{d}^{3} r \hat{\mathbf{P}}_{\mathrm{A}}(\mathbf{r}) \hat{\mathbf{P}}_{\mathrm{A}}(\mathbf{r})-\int \mathrm{d}^{3} r \hat{\mathbf{P}}_{\mathrm{A}}(\mathbf{r}) \hat{\mathbf{E}}_{\mathrm{M}}(\mathbf{r}),(55)
\end{aligned}
$$

where $\hat{\mathbf{B}}(\mathbf{r})=\boldsymbol{\nabla} \times \hat{\mathbf{A}}(\mathbf{r})$ with $\hat{\mathbf{A}}(\mathbf{r})$ from Eq. (6) [together with Eqs. (8) and (9)], and $\hat{\mathbf{E}}_{\mathrm{M}}(\mathbf{r})$ is defined by Eq. (16) [together with Eq. (9)]. Note that in the multipolarcoupling scheme the operator of the electric field strength is defined according to

$$
\begin{aligned}
\hat{\mathbf{E}}(\mathbf{r}) & =-\frac{1}{i \hbar}[\hat{\mathbf{A}}(\mathbf{r}), \hat{\mathcal{H}}]-\nabla \hat{\varphi}_{\mathrm{M}}(\mathbf{r})-\nabla \hat{\varphi}_{\mathrm{A}}(\mathbf{r}) \\
& =\hat{\mathbf{E}}_{\mathrm{M}}(\mathbf{r})-\frac{1}{\varepsilon_{0}} \hat{\mathbf{P}}_{\mathrm{A}}(\mathbf{r}),
\end{aligned}
$$

i.e.,

$$
\varepsilon_{0} \hat{\mathbf{E}}_{\mathrm{M}}(\mathbf{r})=\varepsilon_{0} \hat{\mathbf{E}}(\mathbf{r})+\hat{\mathbf{P}}_{\mathrm{A}}(\mathbf{r})
$$

Hence, $\varepsilon_{0} \hat{\mathbf{E}}_{\mathrm{M}}(\mathbf{r})$ has the meaning of the displacement field with respect to the polarization of the atomic system.

In the electric-dipole approximation, Eq. (55) simplifies to

$$
\hat{\mathcal{H}}=\hat{\mathcal{H}}_{\mathrm{F}}+\hat{\mathcal{H}}_{\mathrm{A}}+\hat{\mathcal{H}}_{\mathrm{AF}}
$$

where

$$
\hat{\mathcal{H}}_{\mathrm{F}}=\int \mathrm{d}^{3} r \int_{0}^{\infty} \mathrm{d} \omega \hbar \omega \hat{\mathbf{f}}^{\dagger}(\mathbf{r}, \omega) \hat{\mathbf{f}}(\mathbf{r}, \omega)
$$

and

$$
\hat{\mathcal{H}}_{\mathrm{A}}=\sum_{\alpha} \frac{\hat{\mathbf{p}}_{\alpha}^{2}}{2 m_{\alpha}}+\frac{1}{2 \varepsilon_{0}} \int \mathrm{d}^{3} r \hat{\mathbf{P}}_{\mathrm{A}}(\mathbf{r}) \hat{\mathbf{P}}_{\mathrm{A}}(\mathbf{r}),
$$

respectively, are the unperturbed Hamiltonians of the medium-assisted electromagnetic field and the atomic system, and

$$
\hat{\mathcal{H}}_{\mathrm{AF}}=-\hat{\mathbf{d}} \hat{\mathbf{E}}_{\mathrm{M}}\left(\mathbf{r}_{\mathrm{A}}\right)
$$

is the interaction energy between them, where $\hat{\mathbf{d}}$ is the atomic dipole operator given by Eq. (29). Recall that $\hat{\mathbf{E}}_{\mathrm{M}}\left(\mathbf{r}_{\mathrm{A}}\right)$ must be thought of a being expressed in terms of the fundamental field variables $\hat{\mathbf{f}}\left(\mathbf{r}_{\mathrm{A}}\right)$ [and $\hat{\mathbf{f}}^{\dagger}\left(\mathbf{r}_{\mathrm{A}}\right)$ ]. Comparing Eq. (60) with Eq. (23), we see that, on taking into account the the relationship

$$
\frac{1}{2} \int \mathrm{d}^{3} r \hat{\rho}_{\mathrm{A}}(\mathbf{r}) \hat{\varphi}_{\mathrm{A}}(\mathbf{r})=\frac{1}{2 \varepsilon_{0}} \int \mathrm{d}^{3} r \hat{\mathbf{P}}_{\mathrm{A}}^{\|}(\mathbf{r}) \hat{\mathbf{P}}_{\mathrm{A}}^{\|}(\mathbf{r}),
$$


the atomic Hamiltonians $\hat{\mathcal{H}}_{\mathrm{A}}$ and $\hat{H}_{\mathrm{A}}$ are different from each other, so that the solution of the eigenvalue problem

$$
\hat{\mathcal{H}}_{\mathrm{A}}\left|n^{\prime}\right\rangle=E_{n}^{\prime}\left|n^{\prime}\right\rangle
$$

may be different from that defined by Eq. (25). Keeping in mind this difference, we drop the primes denoting the atomic eigenvalues and eigenstates from here on.

In contrast to the interaction energy in the minimalcoupling scheme, Eq. (26), the interaction energy in the multipolar-coupling scheme, Eq. (61), is linear in $\hat{\mathbf{f}}\left(\mathbf{r}_{\mathrm{A}}\right)$ and $\hat{\mathbf{f}}^{\dagger}\left(\mathbf{r}_{\mathrm{A}}\right)$. As a consequence of the latter, there is no first-order correction to the ground state energy. We thus have

$$
\Delta E \simeq \Delta_{2} E
$$

where

$$
\begin{aligned}
\Delta_{2} E= & \sum_{n} \int_{0}^{\infty} \mathrm{d} \omega \int \mathrm{d}^{3} r \\
& \times \frac{\left|\left\langle 0\left|\left\langle\{0\}\left|\hat{\mathbf{d}} \hat{\mathbf{E}}_{\mathrm{M}}(\mathbf{r})\right|\{\mathbf{1}(\mathbf{r}, \omega)\}\right\rangle\right| n\right\rangle\right|^{2}}{E_{0}-\left(E_{n}+\hbar \omega\right)}
\end{aligned}
$$

[cf. the first line in Eq. (32) with $\hat{\mathcal{H}}_{A F}$ instead of $\hat{H}_{\mathrm{AF}}^{(\mathrm{I})}$ ]. In complete analogy to the derivation of Eq. (40), we find that

$$
\Delta_{2} E=-\frac{\mu_{0}}{\pi} \sum_{n} \int_{0}^{\infty} \mathrm{d} \omega \frac{\omega^{2}}{\omega_{n}+\omega} \mathbf{d}_{0 n} \operatorname{Im} \boldsymbol{G}\left(\mathbf{r}_{\mathrm{A}}, \mathbf{r}_{\mathrm{A}}, \omega\right) \mathbf{d}_{n 0}
$$

To further evaluate the $\mathbf{r}_{\mathrm{A}}$-dependent part $U\left(\mathbf{r}_{\mathrm{A}}\right)=$ $\Delta_{2}^{(1)} E\left(\mathbf{r}_{\mathrm{A}}\right)$ of $\Delta_{2} E$, which results from the scattering part of the Green tensor, $\boldsymbol{G}^{(1)}\left(\mathbf{r}_{\mathrm{A}}, \mathbf{r}_{\mathrm{A}}, \boldsymbol{\omega}\right)$, and gives the vdW energy, we again write $\operatorname{Im} \boldsymbol{G}^{(1)}=\left(\boldsymbol{G}^{(1)}-\boldsymbol{G}^{(1) *}\right) /(2 i)$, use the relation (12), and change the integration variable from $-\omega$ to $\omega$. After some algebra we arrive at

$$
\begin{aligned}
U\left(\mathbf{r}_{\mathrm{A}}\right)= & -\frac{\mu_{0}}{2 i \pi} \sum_{n} \mathbf{d}_{0 n}\left[\int_{0}^{\infty} \mathrm{d} \omega \frac{\omega^{2}}{\omega_{n}+\omega} \boldsymbol{G}^{(1)}\left(\mathbf{r}_{\mathrm{A}}, \mathbf{r}_{\mathrm{A}}, \omega\right)\right. \\
& \left.-\int_{-\infty}^{0} \mathrm{~d} \omega \frac{\omega^{2}}{\omega_{n}-\omega} \boldsymbol{G}^{(1)}\left(\mathbf{r}_{\mathrm{A}}, \mathbf{r}_{\mathrm{A}}, \omega\right)\right] \mathbf{d}_{n 0} .
\end{aligned}
$$

As we already know, the integrands of the two frequency integrals appearing in Eq. (67) are analytic functions in the upper half of the complex frequency plane, including the real axis [cf. Eq. (B7)]. We therefore can apply contour integral techniques in a similar way as in the derivation of Eq. (47) from Eq. (46). It is not difficult to see that the result reads

$$
U\left(\mathbf{r}_{\mathrm{A}}\right)=\frac{\mu_{0}}{\pi} \sum_{n} \int_{0}^{\infty} \mathrm{d} u \frac{\omega_{n} u^{2}}{\omega_{n}^{2}+u^{2}} \mathbf{d}_{0 n} \boldsymbol{G}^{(1)}\left(\mathbf{r}_{\mathrm{A}}, \mathbf{r}_{\mathrm{A}}, i u\right) \mathbf{d}_{n 0}
$$

which has exactly the same form as the minimal-coupling result (47), so that it can also be given in the form of Eq. (49). Recall that the values of $\omega_{n}$ and $\mathbf{d}_{0 n}$ obtained in the minimal-coupling scheme may be different from those obtained in the multipolar-coupling scheme, because of the somewhat different eigenvalue equations (25) and (63).

\section{APPLICATION: AN ATOM NEAR A SPHERE}

Let us apply the theory to an atom near a dispersing and absorbing dielectric (micro-)sphere surrounded by vacuum. The material of the sphere of radius $R$ is assumed to be homogeneous and isotropic, having a permittivity $\varepsilon(\omega)$. The coordinate system is chosen such that its origin lies at the center of the sphere. The scattering Green tensor can be given by 38$]$

$$
\begin{gathered}
\boldsymbol{G}^{(1)}\left(\mathbf{r}_{\mathrm{A}}, \mathbf{r}_{\mathrm{A}}, i u\right)=\frac{u}{4 \pi c} \sum_{n=1}^{\infty} \sum_{m=0}^{n}\left(2-\delta_{m 0}\right) \frac{2 n+1}{n(n+1)} \\
\times \frac{(n-m) !}{(n+m) !}\left[B_{n}^{M} \sum_{p=-1,1} \mathbf{M}_{n m, p}\left(\mathbf{r}_{\mathrm{A}}\right) \otimes \mathbf{M}_{n m, p}\left(\mathbf{r}_{\mathrm{A}}\right)\right. \\
\left.+B_{n}^{N} \sum_{p=-1,1} \mathbf{N}_{n m, p}\left(\mathbf{r}_{\mathrm{A}}\right) \otimes \mathbf{N}_{n m, p}\left(\mathbf{r}_{\mathrm{A}}\right)\right]
\end{gathered}
$$

where $\mathbf{M}_{n m, p}\left(\mathbf{r}_{\mathrm{A}}\right)$ and $\mathbf{N}_{n m, p}\left(\mathbf{r}_{\mathrm{A}}\right)$ are even $(p=1)$ and odd $(p=-1)$ spherical wave vector functions, which can be expressed in terms of spherical Hankel functions of the first kind, $h_{n}^{(1)}(r)$, and associated Legendre functions, $P_{n}^{m}(\cos \theta)$, in the following way:

$$
\begin{aligned}
& \mathbf{M}_{n m, \pm 1}\left(\mathbf{r}_{\mathrm{A}}\right)=\mp \frac{m}{\sin (\theta)} h_{n}^{(1)}\left(k_{0} r_{\mathrm{A}}\right) P_{n}^{m}(\cos \theta) \\
& \times{ }_{\cos }^{\sin }(m \phi) \mathbf{e}_{\theta}-h_{n}^{(1)}\left(k_{0} r_{\mathrm{A}}\right) \frac{\mathrm{d} P_{n}^{m}(\cos \theta)}{\mathrm{d} \theta} \sin _{\sin }^{\cos }(m \phi) \mathbf{e}_{\phi}, \\
& \mathbf{N}_{n m, \pm 1}\left(\mathbf{r}_{\mathrm{A}}\right)=n(n+1) \frac{h_{n}^{(1)}\left(k_{0} r_{\mathrm{A}}\right)}{k_{0} r_{\mathrm{A}}} P_{n}^{m}(\cos \theta) \\
& \times \sin _{\sin }^{\cos }(m \phi) \mathbf{e}_{r}+\frac{1}{k_{0} r_{\mathrm{A}}} \frac{\mathrm{d}\left[r_{\mathrm{A}} h_{n}^{(1)}\left(k_{0} r_{\mathrm{A}}\right)\right]}{\mathrm{d} r_{\mathrm{A}}}\left[\frac{\mathrm{d} P_{n}^{m}(\cos \theta)}{\mathrm{d} \theta}\right. \\
& \left.\times{ }_{\sin }^{\cos }(m \phi) \mathbf{e}_{\theta} \mp \frac{m}{\sin \theta} P_{n}^{m}(\cos \theta) \underset{\cos }{\sin }(m \phi) \mathbf{e}_{\phi}\right] \text {. }
\end{aligned}
$$

Here, $k_{0}=\omega / c=i u / c$ is the vacuum wave number, and $\mathbf{e}_{r}, \mathbf{e}_{\theta}, \mathbf{e}_{\phi}$, are the mutually orthogonal unit vectors pointing in the directions of $r, \theta$, and $\phi$, respectively. The coefficients $B_{n}^{M}$ and $B_{n}^{N}$ in Eq. (69) read

$$
\begin{aligned}
B_{n}^{M} & =B_{n}^{M}(i u) \\
& =-\frac{\left[z_{1} j_{n}\left(z_{1}\right)\right]^{\prime} j_{n}\left(z_{0}\right)-\left[z_{0} j_{n}\left(z_{0}\right)\right]^{\prime} j_{n}\left(z_{1}\right)}{\left[z_{1} j_{n}\left(z_{1}\right)\right]^{\prime} h_{n}^{(1)}\left(z_{0}\right)-\left[z_{0} h_{n}^{(1)}\left(z_{0}\right)\right]^{\prime} j_{n}\left(z_{1}\right)},
\end{aligned}
$$




$$
\begin{aligned}
B_{n}^{N} & =B_{n}^{N}(i u) \\
& =-\frac{\varepsilon(i u) j_{n}\left(z_{1}\right)\left[z_{0} j_{n}\left(z_{0}\right)\right]^{\prime}-j_{n}\left(z_{0}\right)\left[z_{1} j_{n}\left(z_{1}\right)\right]^{\prime}}{\varepsilon(i u) j_{n}\left(z_{1}\right)\left[z_{0} h_{n}^{(1)}\left(z_{0}\right)\right]^{\prime}-h_{n}^{(1)}\left(z_{0}\right)\left[z_{1} j_{n}\left(z_{1}\right)\right]^{\prime}},
\end{aligned}
$$

where $z_{0}=k_{0} R$ and $z_{1}=k R$, with $k=k_{0} \sqrt{\varepsilon(i u)}$ being the wave number inside the sphere, and $j_{n}(z)$ is the spherical Bessel function of the first kind. The primes indicate differentiations with respect to $z_{0}$ or $z_{1}$, respectively. The coefficients $B_{n}^{M}$ represent contributions from transverse electric (TE) waves reflected at the surface of the sphere, while the coefficients $B_{n}^{N}$ represent those from transverse magnetic (TM) waves.

Substituting the trace of $\boldsymbol{G}^{(1)}\left(\mathbf{r}_{\mathrm{A}}, \mathbf{r}_{\mathrm{A}}, \omega\right)$ from Eq. (69) [together with Eqs. (70) and (71)] into Eq. (150) yields the vdW energy sought. The sums over $p$ can then easily be performed using the orthogonality of the unit vectors $\mathbf{e}_{r}$, $\mathbf{e}_{\theta}$, and $\mathbf{e}_{\phi}$, and the sum over $m$ can be performed with the aid of the summation formulas in Appendix [C So after a lengthy, but straightforward calculation we arrive at the following result:

$$
\begin{aligned}
& U\left(\mathbf{r}_{\mathrm{A}}\right)=-\frac{\hbar \mu_{0}}{8 \pi^{2} c} \int_{0}^{\infty} \mathrm{d} u\left(u^{3} \alpha(i u) \sum_{n=1}^{\infty}(2 n+1)\right. \\
& \times\left\{B_{n}^{M}\left[h_{n}^{(1)}\left(k_{0} r_{\mathrm{A}}\right)\right]^{2}+n(n+1) B_{n}^{N}\left[\frac{h_{n}^{(1)}\left(k_{0} r_{\mathrm{A}}\right)}{k_{0} r_{\mathrm{A}}}\right]^{2}\right. \\
& \left.\left.\quad+B_{n}^{N}\left[\frac{1}{k_{0} r_{\mathrm{A}}} \frac{\mathrm{d}\left[r_{\mathrm{A}} h_{n}^{(1)}\left(k_{0} r_{\mathrm{A}}\right)\right]}{\mathrm{d} r_{\mathrm{A}}}\right]^{2}\right\}\right)
\end{aligned}
$$

Note that the vdW potential does not depend on the angle variables of the atomic position, but only on the distance of the atom from the center of the sphere, as can be anticipated from the symmetry of the system. Recall that the terms proportional to $B_{n}^{M(N)}$ represent the contributions from the TE (TM) waves. Equation (74) applies to an arbitrary dielectric sphere. In particular, when material absorption is omitted, then the result in Ref. [22] can be recovered.

\section{Long-distance limit}

A detailed analysis of Eq. (74) requires numerical computation. Here, however, we would like to focus our attention on two interesting limiting cases, where the atom is very far from or very close to the sphere. Let us first consider the limit of the atom being far away from the sphere,

$$
r_{\mathrm{A}} \gg R
$$

In this case, Eq. (74) reduces to

$$
\begin{aligned}
U\left(\mathbf{r}_{\mathrm{A}}\right) \simeq-\frac{\hbar c R^{3}}{4 \pi^{2} \varepsilon_{0}} \frac{1}{r_{\mathrm{A}}^{7}} \int_{0}^{\infty} \mathrm{d} z \alpha\left(i c z / r_{\mathrm{A}}\right) \frac{\varepsilon\left(i c z / r_{\mathrm{A}}\right)-1}{\varepsilon\left(i c z / r_{\mathrm{A}}\right)+2} \\
\times\left[2(1+z)^{2}+\left(1+z+z^{2}\right)^{2}\right] e^{-2 z}
\end{aligned}
$$

(see Appendix D), where it turns out that the TE waves do not contribute. Since the inequalities $\varepsilon\left(i c z / r_{\mathrm{A}}\right)>1$ and $\alpha\left(i c z / r_{\mathrm{A}}\right)>0$ are valid, the vdW potential is negative, and the resulting force between the atom and the sphere is attractive.

As is seen from Eq. (76), the main contribution to the integral comes from the region where $z \lesssim 1 / 2$. Therefore, for sufficiently large distances, the contributions from small frequencies dominate, and we can (approximately) replace the atomic polarizability and the material permittivity in Eq. (76) with their static values $\alpha^{(0)}=\alpha(\omega=0)$ and $\varepsilon^{(0)}=\varepsilon(\omega=0)$, respectively. The integration can then be performed in closed form to yield the asymptotic distance law

$$
U\left(\mathbf{r}_{\mathrm{A}}\right)=-\frac{23 \hbar c R^{3} \alpha^{(0)}}{16 \pi^{2} \varepsilon_{0}} \frac{\varepsilon^{(0)}-1}{\varepsilon^{(0)}+2} \frac{1}{r_{\mathrm{A}}^{7}} \quad\left(\frac{r_{A}}{R} \rightarrow \infty\right),
$$

in this so-called retarded limit. Note that in the opposite nonretarded limit, where the contributions of $\alpha(\omega)$ and $\varepsilon(\omega)$ at all frequencies have to be retained, Eq. (76) reduces to the result given in Ref. [22], where a $r_{\mathrm{A}}^{-6}$ law was found. The (formal) limit $\varepsilon^{(0)} \rightarrow \infty$ in Eq. (77) obviously corresponds to a metallic sphere

$$
U\left(\mathbf{r}_{\mathrm{A}}\right)=-\frac{23 \hbar c R^{3} \alpha^{(0)}}{16 \pi^{2} \varepsilon_{0}} \frac{1}{r_{\mathrm{A}}^{7}} \quad\left(\frac{r_{A}}{R} \rightarrow \infty\right) .
$$

Note that the decrease of the force with the distance is of three powers stronger than in the case of the atom being near a planar body.

In particular, if we introduce the static polarizability of the sphere (see, e.g., 39])

$$
\alpha_{\mathrm{sph}}^{(0)}=4 \pi \varepsilon_{0} \frac{\varepsilon^{(0)}-1}{\varepsilon^{(0)}+2} R^{3},
$$

we may rewrite Eq. (77) as

$$
U\left(\mathbf{r}_{\mathrm{A}}\right)=-\frac{\alpha_{\mathrm{sph}}^{(0)} \alpha^{(0)}}{\left(4 \pi \varepsilon_{0}\right)^{2}} \frac{23 \hbar c}{4 \pi} \frac{1}{r_{\mathrm{A}}^{7}} \quad\left(\frac{r_{A}}{R} \rightarrow \infty\right) .
$$

Interestingly, Eq. 80 also applies to the vdW potential between two atoms [3], if the (static) polarizability of the sphere is replaced with the polarizability of the second atom.

\section{Short-distance limit}

Let us now proceed to the short-distance limit of the atom being located at a position very close to the sphere, 
i.e.,

$$
\frac{\Delta r_{\mathrm{A}}}{R} \ll 1
$$

$\left(\Delta r_{\mathrm{A}} \equiv r_{\mathrm{A}}-R\right)$. In this case, from Eq. (74) it follows that

$$
U\left(\mathbf{r}_{\mathrm{A}}\right) \simeq-\frac{\hbar}{16 \pi^{2} \varepsilon_{0}} \frac{1}{\left(\Delta r_{\mathrm{A}}\right)^{3}} \int_{0}^{\infty} \mathrm{d} u \alpha(i u) \frac{\varepsilon(i u)-1}{\varepsilon(i u)+1}
$$

(see Appendix E). Note that again the TE waves do not contribute to $U\left(\mathbf{r}_{\mathrm{A}}\right)$.

As expected, the dependence on distance of the (attractive) vdW potential corresponds to that obtained in the case of the atom being near a planar body. In fact, it exactly looks like that derived in Ref. [20] for an atom in the vicinity of a planar, semi-infinite, non-absorbing dielectric. In particular, if the (model) assumption $[\varepsilon(i u)$ $-1] /[\varepsilon(i u)+1]=1$ were made for all values of $u$, then Eq. (82) would lead, on using Eq. (51), to the result [3]

$$
U\left(\mathbf{r}_{\mathrm{A}}\right) \simeq-\frac{\left\langle 0\left|\hat{\mathbf{d}}^{2}\right| 0\right\rangle}{48 \pi \varepsilon_{0}} \frac{1}{\left(\Delta r_{\mathrm{A}}\right)^{3}} .
$$

\section{Material absorption}

To explore the effect of material absorption, we may assume a permittivity of Drude-Lorentz type,

$$
\varepsilon(\omega)=1+\sum_{l} \frac{\Omega_{l}^{2}}{\omega_{l}^{2}-\omega^{2}-i \omega \gamma_{l}},
$$

where $\omega_{l}$ and $\gamma_{l}$ are respectively the (transverse) resonance frequencies and the associated absorption constants, and the frequencies $\Omega_{l}$ are proportional to the so-called oscillator strengths. From Eq. (84) it is seen that in the limit $\omega \rightarrow 0$ the resulting static permittivity

$$
\varepsilon^{(0)}=1+\sum_{l} \frac{\Omega_{l}^{2}}{\omega_{l}^{2}}
$$

is independent of the absorption parameters. Since it is the static permittivity that enters Eq. (77), we see that the long-distance asymptote of the vdW potential is not influenced by material absorption.

With decreasing distance the range of frequency that must be taken into account increases. Thus, the frequency response of the permittivity becomes crucial to the strength of the vdW force. Let us consider the shortdistance law (82). From Eq. (84) it follows that

$$
\frac{\partial}{\partial \gamma_{l}} \frac{\varepsilon(i u)-1}{\varepsilon(i u)+1}=-\frac{1}{[\varepsilon(i u)+1]^{2}} \frac{2 u \Omega_{l}^{2}}{\left(\omega_{l}^{2}+u^{2}+u \gamma_{l}\right)^{2}},
$$

that is to say,

$$
\frac{\partial}{\partial \gamma_{l}} \frac{\varepsilon(i u)-1}{\varepsilon(i u)+1}<0 \quad \text { if } \quad u>0
$$

With regard to Eq. (82), we therefore find that, on recalling that $\alpha(i u)>0$,

$$
\frac{\partial}{\partial \gamma_{l}}\left|\frac{\partial U\left(\mathbf{r}_{\mathrm{A}}\right)}{\partial r_{\mathrm{A}}}\right|<0
$$

Hence, the vdW force monotonically decreases with increasing absorption constants.

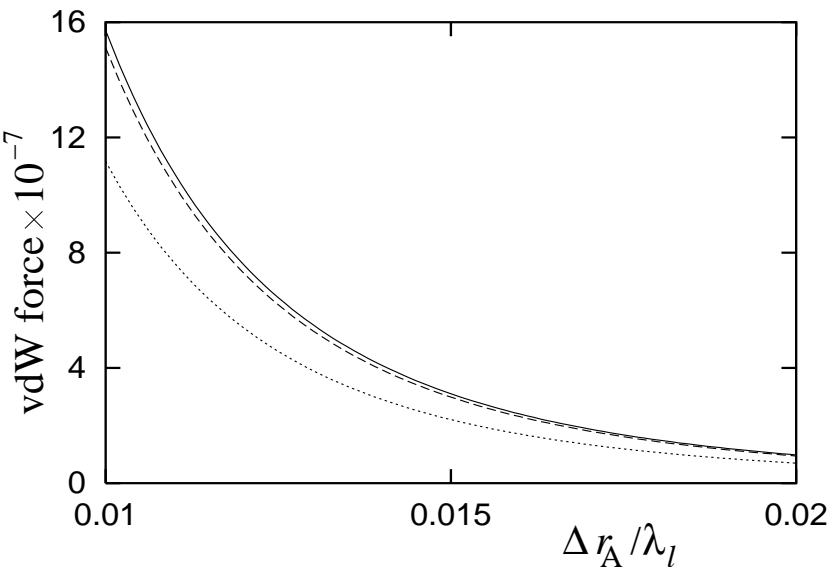

FIG. 1: Absolute value of the normalized van der Waals force $C\left|\partial U\left(\mathbf{r}_{\mathrm{A}}\right) / \partial r_{\mathrm{A}}\right|\left[C=16 \pi^{2} \varepsilon_{0} /\left(\sum_{n}\left|\mathbf{d}_{0 n}\right|^{2} \lambda_{l}^{4}\right), \lambda_{l}=2 \pi c / \Omega_{l}\right]$ as a function of the atom-surface distance for various strengths of material absorption. In the calculation, a metallic permittivity of Drude type according to Eq. 84) and a (degenerate) single-resonance atomic polarizability are assumed with $\omega_{l}=0, \omega_{n} / \Omega_{l}=7 \times 10^{-1}$, and $\gamma_{l} / \Omega_{l}=10^{-2}$ (solid line), $\gamma_{l} / \Omega_{l}=10^{-1}$ (dashed line), and $\gamma_{l} / \Omega_{l}=1$ (dotted line).

Figure円illustrates the influence of material absorption on the vdW force acting on an atom located near a metallic sphere in the short-distance limit. It can be seen that the effect of material absorption increases with decreasing atom-surface distance. In particular, at a distance of $\Delta r_{\mathrm{A}} \simeq 10^{-2} \lambda_{l}$, an increase of the relative absorption parameter from $\gamma_{l} / \Omega_{l}=10^{-2}$ to $\gamma_{l} / \Omega_{l}=1$ would reduce the magnitude of the force by nearly thirty percents.

\section{CONCLUSIONS}

Within the frame of macroscopic QED, we have derived an expression for the $\mathrm{vdW}$ potential of an atomic system near an arbitrary configuration of dispersing and absorbing bodies. It generalizes the results obtained by means of normal-mode expansion and may be regarded as a foundation of the results inferred from linear response theory. We have performed the calculations for both the minimal-coupling scheme and the multipolarcoupling scheme and shown that the results are essentially the same.

We have applied the theory to the vdW interaction between an atom and a sphere. From the integral expression, we have derived the correct long-distance law 
corresponding to the retardation limit and recovered the short-distance law corresponding to the non-retardation limit. In particular, replacing in the long-distance law the polarizability of the sphere with that of an atom just yields the vdW potential between two atoms. On the other hand, for sufficiently small distances of the atom from the sphere the vdW potential approaches the potential observed for an atom near a planar body.

It is worth noting that in the long-distance limit it is the static permittivity that enters the vdW potential. Hence material absorption has no effect on it. However, with decreasing distance of the atom from the sphere the relevant frequencies extend for a finite (increasing) interval and material absorption becomes substantial, thereby diminishing the strength of the force.

In this article, we have restricted our attention to ground-state systems and calculated the vdW potential in lowest-order of perturbation theory with respect to the interaction of the atomic system with the mediumassisted electromagnetic field. The theory allows of course extensions in several respects. As a consequence of the lowest-order perturbation theory, the energy denominators that enter the polarizability of the atomic system are the unperturbed ones, without consideration of the level shift and broadening caused by the presence of the bodies. In fact, the polarizability of an atomic system is expected to drastically change when it becomes close to a macroscopic body and the spontaneous decay thus becomes purely radiationless, with the decay rate being proportional to $\Delta r_{\mathrm{A}}^{-3}$ [32]. Since the level broadening is essentially determined by the spontaneous-decay rate, the polarizability becomes distance-dependent - an effect that needs careful consideration.

Since the electromagnetic field in (linear) magnetic media can be quantized analogously [35], another interesting extension of the theory be the inclusion in it of composite materials characterized by both a complex permittivity and a complex permeability. Interestingly, such materials, which have been fabricated recently, are left-handed. Last not least the underlying quantization scheme renders it also possible to extend the theory to atoms and molecules in excited states and treat the motion of driven atomic systems.

\section{Acknowledgments}

We would like to thank Ludwig Knöll for valuable discussions. This work was supported by the Deutsche Forschungsgemeinschaft.

\section{APPENDIX A: DERIVATION OF EQ. (37)}

From the commutation relation

$$
\left[\hat{r}_{\alpha, i}, \hat{H}_{A}\right]=\left[\hat{r}_{\alpha, i}, \sum_{\beta} \frac{\hat{\mathbf{p}}_{\beta}^{2}}{2 m_{\beta}}\right]=\frac{i \hbar}{m_{\alpha}} \hat{p}_{\alpha, i}
$$

together with the eigenvalue equation (25) we find that

$$
\begin{aligned}
\left\langle 0\left|\hat{p}_{\alpha, i}\right| n\right\rangle & =-\frac{i m_{\alpha}}{\hbar}\left\langle 0\left|\left[\hat{r}_{\alpha, i}, \hat{H}_{A}\right]\right| n\right\rangle \\
& =-\frac{i m_{\alpha}}{\hbar}\left\langle 0\left|\hat{r}_{\alpha, i} \hat{H}_{A}-\hat{H}_{A} \hat{r}_{\alpha, i}\right| n\right\rangle \\
& =-\frac{i m_{\alpha}}{\hbar}\left(E_{n}-E_{0}\right)\left\langle 0\left|\hat{r}_{\alpha, i}\right| n\right\rangle \\
& =-i m_{\alpha} \omega_{n}\left\langle 0\left|\hat{r}_{\alpha, i}\right| n\right\rangle .
\end{aligned}
$$

Thus

$$
\begin{aligned}
\sum_{\alpha} \frac{q_{\alpha}}{m_{\alpha}}\left\langle 0\left|\hat{p}_{\alpha, i}\right| n\right\rangle & =-i \omega_{n} \sum_{\alpha} q_{\alpha}\left\langle 0\left|\hat{r}_{\alpha, i}\right| n\right\rangle \\
& =-i \omega_{n}\left\langle 0\left|\hat{d}_{i}\right| n\right\rangle .
\end{aligned}
$$

Using Eq. A3), we derive

$$
\begin{gathered}
\frac{1}{2 \hbar} \sum_{n} \omega_{n}\left(\left\langle 0\left|\hat{d}_{i}\right| n\right\rangle\left\langle n\left|\hat{d}_{j}\right| 0\right\rangle+\left\langle 0\left|\hat{d}_{j}\right| n\right\rangle\left\langle n\left|\hat{d}_{i}\right| 0\right\rangle\right) \\
=\frac{i}{2 \hbar} \sum_{\alpha} \frac{q_{\alpha}}{m_{\alpha}} \sum_{n}\left(\left\langle 0\left|\hat{p}_{\alpha, i}\right| n\right\rangle\left\langle n\left|\hat{d}_{j}\right| 0\right\rangle\right. \\
\left.\quad-\left\langle 0\left|\hat{d}_{j}\right| n\right\rangle\left\langle n\left|\hat{p}_{\alpha, i}\right| 0\right\rangle\right) \\
=\frac{i}{2 \hbar} \sum_{\alpha} \frac{q_{\alpha}}{m_{\alpha}}\left\langle 0\left|\left[\hat{p}_{\alpha, i}, \hat{d}_{j}\right]\right| 0\right\rangle \\
=\frac{i}{2 \hbar} \sum_{\alpha} \frac{q_{\alpha}}{m_{\alpha}}\left\langle 0\left|\left[\hat{p}_{\alpha, i}, \sum_{\beta} q_{\beta} \hat{r}_{\beta, j}\right]\right| 0\right\rangle \\
=\sum_{\alpha} \frac{q_{\alpha}^{2}}{2 m_{\alpha}} \delta_{i j},
\end{gathered}
$$

which is just Eq. (37).

\section{APPENDIX B: ASYMPTOTIC BEHAVIOR OF THE GREEN TENSOR}

The asymptotic behavior of the Green tensor for large frequencies reads [35]

$$
\begin{gathered}
\lim _{|\omega| \rightarrow \infty} \frac{\omega^{2}}{c^{2}} \boldsymbol{G}\left(\mathbf{r}, \mathbf{r}^{\prime}, \omega\right)=-\boldsymbol{\delta}\left(\mathbf{r}-\mathbf{r}^{\prime}\right), \\
\lim _{|\omega| \rightarrow \infty} \frac{\omega^{2}}{c^{2}} \boldsymbol{G}^{(0)}\left(\mathbf{r}, \mathbf{r}^{\prime}, \omega\right)=-\boldsymbol{\delta}\left(\mathbf{r}-\mathbf{r}^{\prime}\right) .
\end{gathered}
$$

If $\mathbf{r}$ and $\mathbf{r}^{\prime}$ lie in a common region of constant permittivity, we can use Eq. (43), and subtract the two equations (B1) and (B2) to obtain

$$
\lim _{|\omega| \rightarrow \infty} \frac{\omega^{2}}{c^{2}} \boldsymbol{G}^{(1)}\left(\mathbf{r}, \mathbf{r}^{\prime}, \omega\right)=0 .
$$


In the low-frequency limit we have 35]

$$
\lim _{|\omega| \rightarrow 0} \frac{\omega^{2}}{c^{2}} \boldsymbol{G}\left(\mathbf{r}, \mathbf{r}^{\prime}, \omega\right)=-{ }^{\|} \boldsymbol{L}^{-1 \|}\left(\mathbf{r}, \mathbf{r}^{\prime}\right)
$$

where

$$
\boldsymbol{L}\left(\mathbf{r}, \mathbf{r}^{\prime}\right)=\lim _{|\omega| \rightarrow 0} \int \mathrm{d}^{3} s \boldsymbol{\delta}^{\|}(\mathbf{r}-\boldsymbol{s}) \varepsilon(\mathbf{s}, \omega) \boldsymbol{\delta}^{\|}\left(\boldsymbol{s}-\mathbf{r}^{\prime}\right)
$$

Recalling that as $|\omega| \rightarrow 0$,

$$
\varepsilon(\mathbf{r}, \omega) \sim \begin{cases}\omega^{0} & \text { for dielectrics } \\ (i \omega)^{-1} & \text { for metals }\end{cases}
$$

[cf. Eq. (84)], from Eqs. (B4) and (B5) we see that

$$
\lim _{|\omega| \rightarrow 0} \omega^{2} \boldsymbol{G}\left(\mathbf{r}, \mathbf{r}^{\prime}, \omega\right)=M, \quad M<\infty
$$

Needless to say, that Eq. (B7) is also valid for the scattering part of the Green tensor, $\boldsymbol{G}^{(1)}\left(\mathbf{r}, \mathbf{r}^{\prime}, \omega\right)$.

\section{APPENDIX C: SUMMATION FORMULAS FOR LEGENDRE POLYNOMIALS}

The Legendre polynomials obey the relation [40]

$$
\sum_{m=0}^{n} C_{n m} \cos (m \lambda) P_{n}^{m}(x) P_{n}^{m}(y)=P_{n}(\xi)
$$

where

$$
\begin{gathered}
C_{n m}=\left(2-\delta_{m 0}\right) \frac{(n-m) !}{(n+m) !}, \\
\xi \equiv x y+\sqrt{\left(1-x^{2}\right)\left(1-y^{2}\right)} \cos \lambda .
\end{gathered}
$$

For $\lambda=0$ and $x=y=\cos \theta$, Eq. (C1) reduces to

$$
\sum_{m=0}^{n} C_{n m} P_{n}^{m}(\cos \theta)^{2}=1
$$

Differentiating Eq. (C1) twice with respect to $\lambda$ and putting $\lambda=0$ and $x=y=\cos \theta$ afterwards yield

$$
\sum_{m=0}^{n} C_{n m} \frac{m^{2}}{\sin ^{2} \theta} P_{n}^{m}(\cos \theta)^{2}=\frac{n(n+1)}{2} .
$$

Finally, subsequent differentiations of Eq. (C1) with respect to $x$ and $y$ and again putting $\lambda=0$ and $x=y=\cos \theta$ afterwards yield

$$
\sum_{m=0}^{n} C_{n m}\left[\frac{\mathrm{d} P_{n}^{m}(\cos \theta)}{\mathrm{d} \theta}\right]^{2}=\frac{n(n+1)}{2}
$$

\section{APPENDIX D: DERIVATION OF EQ. (76)}

In Eq. (74), the spherical Hankel functions $h_{n}^{(1)}\left(k_{0} r_{\mathrm{A}}\right)$ $=h_{n}^{(1)}\left(\right.$ iur $\left._{\mathrm{A}} / c\right)$ can be written in the form of [40]

$$
h_{n}^{(1)}\left(k_{0} r_{\mathrm{A}}\right)=\sum_{j=0}^{n} h_{j} e^{-u r_{\mathrm{A}} / c}\left(\frac{c}{u r_{\mathrm{A}}}\right)^{j+1}
$$

with some complex coefficients $h_{j}$. From inspection of Eqs. (72) and (73) it is seen that $B_{n}^{M}$ and $B_{n}^{N}$ can be expanded in powers of $u$ at $u=0$,

$$
B_{n}^{M, N}=\sum_{j=0}^{\infty} b_{j}^{M, N}\left(\frac{u R}{c}\right)^{j} .
$$

From Eqs. (D1) and (D2) it then follows that the integrand of the (imaginary) frequency integral in Eq. (74) is a sum of terms, which are all of the same general structure

$$
f_{j k}(u)=\alpha(i u) u^{3}\left(\frac{u R}{c}\right)^{j}\left(\frac{c}{u r_{\mathrm{A}}}\right)^{k+2} e^{-2 u r_{\mathrm{A}} / c}
$$

$(j, k$ are nonnegative integers). For $j>(k-1)$ this is a polynomial in $u$ times an exponentially decaying function. The only relevant contributions to the frequency integral come from the maximum of $f_{j k}(u)$ at a frequency $u_{0}$ satisfying

$$
\left.\frac{\mathrm{d}}{\mathrm{d} u} f_{j k}(u)\right|_{u=u_{o}}=0
$$

thus

$$
u \approx u_{0} \simeq \frac{(j+1-k) c}{2 r_{\mathrm{A}}}
$$

where we have used the fact that $\alpha(i u)$ can be regarded as almost constant for the small frequencies considered here. For $j \leq(k-1), f_{j k}(u)$ is a monotonically decreasing function, and relevant contributions to the frequency integral can only come from regions, where

$$
u \leq \frac{c}{2 r_{\mathrm{A}}}
$$

because for larger frequencies the exponentially decaying factor becomes too small. Combining Eqs. (D5) and (D6), it can be said that the relevant contributions to the frequency integral come from regions, where $u \lesssim c / r_{\mathrm{A}}$. In these regions we have

$$
\left|k_{0} R\right|,|k R| \sim \frac{u R}{c} \lesssim \frac{R}{r_{\mathrm{A}}} .
$$

This means that in the long-distance limit $r_{\mathrm{A}} \gg R$ the main contributions to the integral come from regions, where $\left|k_{0} R\right|,|k R| \ll 1$. We may therefore expand the coefficients $B_{n}^{M, N}$ in Eqs. (72) and (73) in powers of $k_{0} R$, 
on exploiting useful relations in Ref. [40], and retain only the leading terms:

$$
\begin{gathered}
B_{n}^{M}=o\left[\left(k_{0} R\right)^{2 n+3}\right] \\
B_{n}^{N} \simeq i \frac{(n+1)(2 n+1)}{[(2 n+1) ! !]^{2}} \frac{\varepsilon(i u)-1}{\varepsilon(i u) n+n+1}\left(k_{0} R\right)^{2 n+1} .
\end{gathered}
$$

In this way we find that the leading term in Eq. (74) comes from the two terms containing $B_{n}^{N}$ with $n=1$. Keeping only these terms, using [40]

$$
h_{1}^{(1)}(z)=-\left(\frac{1}{z}+\frac{i}{z^{2}}\right) e^{i z}
$$

and changing the integration variable according to $u \rightarrow z$ $=u r_{\mathrm{A}} / c$, we arrive at Eq. (76).

\section{APPENDIX E: DERIVATION OF EQ. (82)}

Provided that

$$
n \gg \frac{|z|^{2}}{4},
$$

the spherical Bessel and Hankel functions appearing in Eqs. (72) - (74) can be approximated by [40]

$$
j_{n}(z) \simeq \frac{z^{n}}{(2 n+1) ! !},
$$

and

$$
h_{n}^{(1)}(z) \simeq-i \frac{(2 n-1) ! !}{z^{(n+1)}},
$$

respectively. As we know from Appendix $D$ the main contribution to the frequency integral in Eq. (74) is from those values satisfying the condition (D6). Hence, the condition (E1) becomes

$$
n \gg 1,
$$

because $z \sim u R / c \simeq u r_{\mathrm{A}} / c$ in the short-distance limit. Substituting in Eqs. (72) - (744) for $j_{n}(z)$ and $h_{n}^{(1)}(z)$ the expressions (E2) and (E3), we derive after some algebra

$$
B_{n}^{M} \simeq 0,
$$

$$
\begin{aligned}
& n(n+1)(2 n+1) B_{n}^{N}\left[\frac{h_{n}^{(1)}\left(k_{0} r_{\mathrm{A}}\right)}{k_{0} r_{\mathrm{A}}}\right]^{2} \\
& +(2 n+1) B_{n}^{N}\left[\frac{\mathrm{d}\left[r_{\mathrm{A}} h_{n}^{(1)}\left(k_{0} r_{\mathrm{A}}\right)\right]}{k_{0} r_{\mathrm{A}} \mathrm{d} r_{\mathrm{A}}}\right]^{2} \\
& \simeq-i \frac{1}{\left(k_{0} r_{\mathrm{A}}\right)^{3}} \frac{\varepsilon(i u)-1}{\varepsilon(i u)+1} n(n+1)\left(\frac{R}{r_{\mathrm{A}}}\right)^{2 n+1} \\
& \quad-i \frac{1}{\left(k_{0} r_{\mathrm{A}}\right)^{3}} \frac{\varepsilon(i u)-1}{\varepsilon(i u)+1} \frac{(2 n+1)^{2}}{4}\left(\frac{R}{r_{\mathrm{A}}}\right)^{2 n+1} \\
& \simeq-2 i \frac{1}{\left(k_{0} r_{\mathrm{A}}\right)^{3}} \frac{\varepsilon(i u)-1}{\varepsilon(i u)+1} n(n+1)\left(\frac{R}{r_{\mathrm{A}}}\right)^{2 n+1}
\end{aligned}
$$

Whereas in the long-distance limit we could neglect all terms but the $n=1$ one (Appendix D), in the shortdistance limit, as can be seen from Eq. (E6), the parameter $R / r_{\mathrm{A}}$ being very close to one, we encounter the opposite extreme, where the main contribution comes from those terms corresponding to high orders $n$. The main contribution to the sum over $n$ in Eq. (74) comes from the peak at $n_{1}$ determined by

$$
\begin{aligned}
& \left.\frac{\mathrm{d}}{\mathrm{d} n}\left[n(n+1)\left(\frac{R}{r_{\mathrm{A}}}\right)^{2 n+1}\right]\right|_{n=n_{1}} \\
& \left.\approx \frac{\mathrm{d}}{\mathrm{d} n}\left(n^{2} e^{2 n\left(\ln R-\ln r_{\mathrm{A}}\right)}\right)\right|_{n=n_{1}} \\
& =2 n_{1}\left(\frac{R}{r_{\mathrm{A}}}\right)^{2 n_{1}}\left[1+n_{1}\left(\ln R-\ln r_{\mathrm{A}}\right)\right]=0,
\end{aligned}
$$

from which we find

$$
n_{1}=\frac{1}{\ln r_{\mathrm{A}}-\ln R} \simeq \frac{R}{\Delta r_{\mathrm{A}}},
$$

because of $\Delta r_{\mathrm{A}} / R \ll 1$. Since the main contribution to the sum over $n$ in Eq. (744) comes from values of $n \approx n_{1} \gg 1$, where the approximate formulas $(\mathrm{E} 5$ ) and (E6) are valid [cf. Eq. (E4)]. Therefore we introduce only a small error if we extrapolate these formulas to the terms with small $n$. Then the sum over $n$ is equal to the second derivative with respect to $\left(R / r_{\mathrm{A}}\right)^{2}$ of a geometric sum, which can be performed in closed form to yield Eq. (82).
[1] G. Binnig, C. F. Quate, and Ch. Gerber, Phys. Rev. Lett. 56, 930 (1986).

[2] F. Shimizu and J. Fujita, Phys. Rev. Lett. 88, 123201 (2002).

[3] H. B. G. Casimir and D. Polder, Phys. Rev. 73, 360 (1948).
[4] D. Raskin and P. Kusch, Phys. Rev. 179, 712 (1969); A. Shih, D. Raskin, and P. Kusch, Phys. Rev. A 9, 652 (1974); A. Shih and V. A. Parsegian, Phys. Rev. A 12, 835 (1975).

[5] A. Anderson, S. Haroche, E. A. Hinds, W. Jhe, and D. Meschede, Phys. Rev. A 37, 3594 (1988); C. I. Sukenik, 
M. G. Boshier, D. Cho, V. Sandoghdar, and E. A. Hinds, Phys. Rev. Lett. 70, 560 (1993).

[6] R. E. Grisenti, W. Schöllkopf, J. P. Toennies, G. C. Hegerfeldt, and T. Köhler, Phys. Rev. Lett. 83, 1755 (1999).

[7] F. Shimizu, Phys. Rev. Lett. 86, 987 (2001); V. Druzhinina and M. DeKieviet, eprint quant-ph/0212076

[8] A. Landragin, J.-Y. Courtois, G. Labeyrie, N. Vansteenkiste, C. I. Westbrook, and A. Aspect, Phys. Rev. Lett. 77, 1464 (1996).

[9] M. Oria, M. Chrevrollier, D. Bloch, M. Fichet, and M. Ducloy, Europhys. Lett. 14, 527 (1991); V. Sandoghdar, C. I. Sukenik, E. A. Hinds, and S. Haroche, Phys. Rev. Lett. 68, 3432 (1992); M. Fichet, F. Schuller, D. Bloch, and M. Ducloy, Phys. Rev. A 51, 1553 (1995); M. Marrocco, M. Weidinger, R. T. Sang, and H. Walther, Phys. Rev. Lett. 81, 5784 (1998); H. Failache, S. Saltiel, M. Fichet, D. Bloch, and M. Ducloy, ibid. 82, 5467 (1999).

[10] M. Gorlicki, S. Feron, V. Lorent, and M. Ducloy, Phys. Rev. A 61, 013603 (1999); R. Marani, L. Cognet, V. Savalli, N. Westbrook, C. I. Westbrook, and A. Aspect, ibid. 61, 053402 (2000).

[11] I. E. Dzyaloshinskii, E. M. Lifshitz, and L. P. Pitaevskii, Adv. Phys. 10, 165 (1961).

[12] D. Langbein, Springer Tracks Mod. Phys. 72, 1 (1974).

[13] J. Mahanty and B. W. Ninham, Dispersion Forces (Academic, London, 1976).

[14] E. A. Hinds, in Advances in Atomic, Molecular, and Optical Physics, edited by D. Bates and B. Bederson (Academic, New York, 1991), Vol. 28, p. 237.

[15] P. W. Milonni, The Quantum Vacuum: An Introduction to Quantum Electrodynamics (Academic, San Diego, 1994).

[16] R. K. Bullough and B. V. Thompson, J. Phys. C 3, 1780 (1970).

[17] M. J. Renne, Physica 53, 193 (1971); ibid. 56, 124 (1971).

[18] P. W. Milonni and M.-L. Shih, Phys. Rev. A 45, 4241 (1992). See also J. Schwinger, L. L. DeRaad, and K. A. Milton, Ann. Phys. (New York) 115, 1 (1978) for a related treatment.

[19] Y. Tikochinski and L. Spruch, Phys. Rev. A 48, 4223 (1993).

[20] F. Zhou and L. Spruch, Phys. Rev. A 52, 297 (1995).

[21] M. Boström and B. E. Sernelius, Phys. Rev. A 61, 052703 (2000). Note that the formulas derived in Ref. [20] for frequency-independent, real permittivities are used for studying metals, by putting complex permittivities in them, without any proof.

[22] A. M. Marvin and F. Toigo, Phys. Rev. A 25, 782 (1982). In fact an energy formula based on a normal-mode expansion is combined with elements of the linear-response theory.

[23] C. H. Wu, C.-I Kuo, and L. H. Ford, Phys. Rev. A 65, 062102 (2002).

[24] A. D. McLachlan, Proc. R. Soc. London Ser. A 271, 387 (1963); Mol. Phys. 7, 381 (1963).

[25] G. S. Agarwal, Phys. Rev. A 11, 243 (1975).

[26] J. M. Wylie and J. E. Sipe, Phys. Rev. A 30, 1185 (1984).

[27] C. Girard, J. Chem. Phys. 85, 6750 (1986).

[28] C. Girard and C. Girardet, J. Chem. Phys. 86, 6531 (1987).

[29] M. Fichet, F. Schuller, D. Bloch, and M. Ducloy, Phys. Rev. A 51, 1553 (1995); M.-P. Gorza, S. Saltiel. H. Failache, and M. Ducloy, Eur. Phys. J. D 15, 113 (2001).

[30] C. Girard, S. Maghezzi, and F. Hache, J. Chem. Phys. 91, 5509 (1989).

[31] M. Boustimi, J. Baudon, P. Candori, and J. Robert, Phys. Rev. B 65, 155402 (2002).

[32] Ho Trung Dung, L. Knöll, and D.-G. Welsch, Phys. Rev. A 64, 013804 (2001); Ho Trung Dung, S. Scheel, L. Knöll, and D.-G. Welsch, J. Opt. B: Quantum Semiclass. Opt. 4, 169 (2002).

[33] J. R. Buck and J. Kimble, Phys. Rev. A 67, 033806 (2003).

[34] S. Scheel, L. Knöll, and D.-G. Welsch, Phys. Rev. A 60, 4094 (1999); Ho Trung Dung, L. Knöll, and D.-G. Welsch, ibid. 62, 053804 (2000).

[35] L. Knöll, S. Scheel, and D.-G. Welsch, in Coherence and Statistics of Photons and Atoms, edited by J. Peřina (John Wiley \& Son, New York, 2001), p. 1.

[36] A. S. Davydov, Quantum Mechanics (NEO, Ann Arbor, MI, 1967), pp.317-319.

[37] Ho Trung Dung, L. Knöll, and D.-G. Welsch, Phys. Rev. A 65, 043813 (2002).

[38] L. W. Li, P. S. Kooi, M. S. Leong, and T. S. Yeo, IEEE Trans. Microwave Theory Tech. 42, 2302 (1994); C.-T. Tai, Dyadic Green Functions in Electromagnetic Theory (IEEE Press, New York, 1994).

[39] J. D. Jackson, Classical Electrodynamics (John Wiley \& Sons, New York, 1998).

[40] Handbook of Mathematical Functions, edited by M. Abramowitz and I. A. Stegun (Dover, New York, 1973). 